\title{
SPACES DETERMINED BY A GROUP OF FUNCTIONS
}

BY C. L. WIGINTON AND SUSAN SHRADER

Communicated by Orville G. Harrold, May 6, 1968

1. Introduction. Let $G_{F}$ denote the group of all homeomorphisms of the topological space $F$ onto itself, and let $G_{F}$, be similarly defined for a space $F^{\prime}$. If $G_{F}$ and $G_{F^{\prime}}$ are topologized under the point open topology, and if there is a function from $G_{F}$ onto $G_{F^{\prime}}$ which is a homeomorphism as well as an algebraic isomorphism then Wechsler [1] has determined a sufficient condition for the spaces $F$ and $F^{\prime}$ to be homeomorphic. Thomas [2] has recently generalized Wechsler's theorem by weakening this condition on the spaces $F$ and $F^{\prime}$. It is the purpose here to generalize Wechsler's theorem in a different direction by using a group of functions other than a group of homeomorphisms.

2. Preliminaries. Most of our notation can be found in [1] and [2]; for reference we include the following. The space $F$ is $n$-homogeneous with respect to a group of functions $G$ provided for any pair of proper $n$-tuples $\left(x_{1}, \cdots, x_{n}\right),\left(y_{1}, \cdots, y_{n}\right)$, there is a $g$ in $G$ such that $g\left(x_{i}\right)=y_{i}, i=1, \cdots, n$. The space $F$ is $\omega$-homogeneous with respect to $a$ group of functions $G$ provided it is $n$-homogeneous with respect to $G$ for each positive integer $n$.

Let $G_{x}=\{f \in G: f(x)=x\}$. Then $G_{x}$ is a subgroup of $G$ and will be called the subgroup of the point $x$. Furthermore $G / G_{x}$ will denote the set of left cosets, and cosets will be written as $f G_{x}$.

We will use the point open topology on $G$ and will consider $G / G_{x}$ to have the topology induced by the natural mapping, that is, $\nu_{x}: G \rightarrow G / G_{x}$ defined by $\nu_{x}(h)=h G_{x}$ is to be continuous so that a set $U$ is open in $G / G_{x}$ if and only if $\nu_{x}^{-1}(U)$ is open in $G$. All spaces are $T_{2}$.

Our main theorem is as follows:

THEOREM 1. Let $F$ be a topological space, and let $G$ denote a group of one-to-one functions from $F$ onto itself with respect to which $F$ is w-homogeneous, and let $F^{\prime}$ and $G^{\prime}$ be similarly defined. Suppose that $\Phi$ is a homeomorphism from $G$ onto $G^{\prime}$ such that $\Phi$ is an isomorphism. Then there is a homeomorphism from $F$ onto $F^{\prime}$.

The proof of the main theorem will be accomplished by showing the existence of a sequence of homeomorphisms whose composition will then be the desired homeomorphism between $F$ and $F^{\prime}$. We prove first that $G / G_{x}$ is homeomorphic to $F$. We then show that $\Phi$ induces a homeomorphism from $G / G_{x}$ onto $G^{\prime} / \Phi\left(G_{x}\right)$. It is next shown that the 
subgroup $\Phi\left(G_{x}\right)$ is, in fact, the subgroup of a point $y$ in $F^{\prime}$, i.e. $\Phi\left(G_{x}\right)$ $=G_{y}^{\prime}$. We have then that $G^{\prime} / \Phi\left(G_{x}\right)=G^{\prime} / G_{y}^{\prime}$. Finally, an application of the first result gives that $G^{\prime} / G_{y}^{\prime}$ is homeomorphic to $F^{\prime}$. Thus, letting $\simeq$ denote the relation of homeomorphism, we have

$$
F \simeq G / G_{x} \simeq G^{\prime} / \Phi\left(G_{x}\right)=G^{\prime} / G_{y}^{\prime} \simeq F^{\prime}
$$

and therefore $F$ is homeomorphic to $F^{\prime}$.

3. The sequence of homeomorphisms. A subbasis element in $G$ is denoted by $W(y, U)=\{f \in G: f(y) \in U\}$ where $y$ is a point of $F$ and $U$ is an open set in $F$. A basis element is denoted by $W(\bar{y}, \bar{U})$ where $\bar{y}=\left(y_{1}, \cdots, y_{n}\right)$ is a proper $n$-tuple and $\bar{U}=\left(U_{1}, \cdots, U_{n}\right)$. The symbol $W(y)$ is used to denote $\{z \in F: g(y)=z, g \in W\}$.

Lemma 1. The function $\mu_{x}: G \rightarrow F$ defined by $\mu_{x}(h)=h(x)$ is continuous and open.

Proof. This is shown in [1] for a group $G$ of homeomorphisms. It is easily seen that the continuity and openness of elements of $G$ are not required.

THEOREM 2. The function $\theta_{x}: G / G_{x} \rightarrow F$ defined by $\theta_{x}\left(h G_{x}\right)=h(x)$ is a homeomorphism.

PRoof. The comment in the proof of Lemma 1 applies here also.

Suppose $F^{\prime}$ is a Hausdorff topological space which is $\omega$-homogeneous with respect to a group $G^{\prime}$ of one-to-one functions from $F^{\prime}$ onto itself. Suppose further that there is a homeomorphism $\Phi$ from $G$ to $G^{\prime}$ which is an isomorphism. Let $H=G_{x}$ and $H^{\prime}=\Phi\left(G_{x}\right)$.

LEMma 2. Using the notation of the previous paragraph, $\Phi$ induces a homeomorphism between $G / H$ and $G^{\prime} / H^{\prime}$ defined as $\phi=\nu^{\prime} \Phi \nu_{x}^{-1}$, where $\nu^{\prime}$ is the natural map from $G^{\prime}$ onto $G^{\prime} / H^{\prime}$.

Proof. Trivial.

Our next task is to show that $H^{\prime}$ is the subgroup of a point $y \in F^{\prime}$. For this we will need several lemmas.

Lemma 3. If $f \in G-H$, then $H f H$ is dense in $G$.

Proof. The proof is contained in Theorem 3.1 of [2] where again the continuity and openness of elements of $G$ are not required.

Lemma 4. $H^{\prime}$ is a proper closed subgroup of $G^{\prime}$.

Proof. Trivial.

Throughout the remainder $W(\bar{x}, \bar{U})$ will denote a nonempty basic open set in $G^{\prime}-H^{\prime}$ such that $\bar{x} \in F^{\prime n}, \bar{U} \subset F^{\prime n}$ and $n$ is the smallest 
integer with this property. If $\bar{w}=\left(w_{1}, \cdots, w_{n}\right)$ denote $\left(w_{1}, \cdots\right.$, $\left.w_{p-1}, w_{p+1}, \cdots, w_{n}\right)$ by $\bar{w}_{p}$.

LEMMA 5. If $n>1$ then $H^{\prime}\left(\bar{x}_{k}\right)$ is dense in $F^{\prime n-1}$ and therefore infinite in each component.

Proof. If there is an open set $O \subset F^{\prime n-1}$ which does not intersect $H^{\prime}\left(\tilde{x}_{k}\right)$, then $W\left(\tilde{x}_{k}, O\right)$ contradicts the minimality of $n$.

LEMMA 6. If $n>1$ there exist two independent points in $H^{\prime}(\bar{x})$.

Proof. The proof is as in Lemma 3.14 of [1].

Lemma 7. Suppose there is an $n$-tuple $\bar{z}$ in $H^{\prime}(\bar{x})$ independent of $\bar{x}$. Then $\bar{x}$ lies in infinitely many distinct sets of the form $g\left(H^{\prime}(\bar{x})\right)$ where $g$ is in $G^{\prime}$.

Proof. This proof is obtained in the same manner as the similar result in [2, Lemma 2.4].

Theorem 3. For some $y$ in $F^{\prime}, H^{\prime}=G_{y}^{\prime}$.

Proof. Having established the preceding Lemmas, the proof given in [2, Theorem 3.1] applies.

4. Proof of Theorem 1. From Theorem 2, we have that $F$ and $G / G_{x}$ are homeomorphic and so also are $F^{\prime}$ and $G^{\prime} / G_{y}^{\prime}$. That $G / G_{x}$ and $G^{\prime} / \Phi\left(G_{x}\right)$ are homeomorphic is a consequence of Lemma 2 . Theorem 3 establishes the equality of $G^{\prime} / \Phi\left(G_{x}\right)$ and $G^{\prime} / G_{y}$. Thus

$$
F \simeq G / G_{x} \simeq G^{\prime} / \Phi\left(G_{x}\right)=G^{\prime} / G_{y}^{\prime} \simeq F^{\prime}
$$

and therefore $F$ and $F^{\prime}$ are homeomorphic.

\section{REFERENCES}

1. M. T. Wechsler, Homeomorphism groups of certain topological spaces, Ann. of Math. 62 (1955), 360-373.

2. E. S. Thomas, Jr., Spaces determined by their homeomorphism groups, Trans. Amer. Math. Soc. 126 (1967), 244-250.

UNIVERSITY OF HoustoN 